\title{
Zearalenone regulates key factors of the Kelch-like erythroid cell-derived protein with CNC homology-associated protein 1 -nuclear factor erythroid 2-related factor 2 signaling pathway in duodenum of post-weaning gilts
}

\author{
Qun Cheng ${ }^{1}$, Shu zhen Jiang ${ }^{1}$, Li bo Huang ${ }^{1}$, Wei ren Yang ${ }^{1 / *}$, and Zai bin Yang ${ }^{1}$
}

\author{
* Corresponding Author: Wei ren Yang \\ Tel: +86-18605489796 \\ Fax: +86-0538-8249371 \\ E-mail:wryang@sdau.edu.cn \\ 1 Shandong Provincial Key Laboratory of \\ Animal Biotechnology and Disease Control \\ and Prevention, Department of Animal \\ Sciences and Technology, Shandong \\ Agricultural University, Taian, Shandong \\ 271018, China \\ ORCID \\ Qun Cheng \\ https://orcid.org/0000-0001-5988-446X \\ Shu zhen Jiang \\ https://orcid.org/0000-0002-4943-0322 \\ Li bo Huang \\ https://orcid.org/0000-0002-5517-8493 \\ Wei ren Yang \\ https://orcid.org/0000-0002-9363-0516 \\ Zai bin Yang \\ https://orcid.org/0000-0001-8757-9120
}

Submitted Jun 2, 2020; Revised Sept 15, 2020; Accepted Sept 25, 2020
Objective: This study explored the mechanism of the Kelch-like erythroid cell-derived protein with CNC homology-associated protein 1 (Keap1)-nuclear factor erythroid 2-related factor 2 (Nrf2) signaling pathway under conditions of zearalenone (ZEA)-induced oxidative stress in the duodenum of post-weaning gilts.

Methods: Forty post-weaning gilts were randomly allocated to four groups and fed diets supplemented with $0,0.5,1.0$, or $1.5 \mathrm{mg} / \mathrm{kg}$ ZEA.

Results: The results showed significant reductions in the activity of the antioxidant enzymes total superoxide dismutase and glutathione peroxidase and increases the malondialdehyde content with increasing concentrations of dietary ZEA. Immunohistochemical analysis supported these findings by showing a significantly increased expression of Nrf2 and glutathione peroxidase 1 (GPX1) with increasing concentrations of ZEA. The relative mRNA and protein expression of Nrf2, GPX1 increased linearly $(\mathrm{p}<0.05)$ and quadratically $(p<0.05)$, which was consistent with the immunohistochemical results. The relative mRNA expression of Keap1 decreased linearly $(\mathrm{p}<0.05)$ and quadratically $(\mathrm{p}<0.05)$ in the duodenum as the ZEA concentration increased in the diet. The relative mRNA expression of modifier subunit of glutamate-cysteine ligase (GCLM) increased quadratically $(p<0.05)$ in all ZEA treatment groups and the relative mRNA expression of quinone oxidoreductase 1 (NQO1) catalytic subunit of glutamate-cysteine ligase decreased linearly $(\mathrm{p}<0.05)$ and quadratically $(\mathrm{p}<0.05)$ in the ZEA1.0 group and ZEA1.5 group. The relative protein expression of Keap 1 and GCLM decreased quadratically $(\mathrm{p}<0.05)$ in the duodenum as the ZEA concentration increased in the diet, respectively. The relative protein expression of NQO1 increased linearly $(\mathrm{p}<0.05)$ and quadratically $(\mathrm{p}<0.05)$ in all ZEA treatment groups in the duodenum.

Conclusion: These findings suggest that ZEA regulates the expression of key factors of the Keap1-Nrf2 signaling pathway in the duodenum, which enables resistance to ZEA-induced oxidative stress. Further studies are needed to examine the effects of ZEA induced oxidative stress on other tissues and organs in post-weaning gilts.

Keywords: Antioxidant; Duodenum; Keap1-Nrf2 Signaling Pathway; Oxidative Stress; Zearalenone

\section{INTRODUCTION}

Zearalenone (ZEA) is a contaminant widely found in cereals and compound feeds that exhibits latent toxicity. This can cause significant economic losses due to reductions in animal production, as well as adversely affecting public health and animal welfare $[1,2]$. Zearalenone, 6-(10-hydroxy-6-oxo-trans-1-undecenyl)- $\beta$-resorcyclic acid lactone, is a 
nonsteroidal estrogen mycotoxin produced by Fusarium fungi $[3,4]$. The structure of ZEA is similar to that of estradiol, which can bind to estrogen receptors in $17 \beta$-estradiol-competitive target cells, resulting in reproductive dysfunction and decreased reproductive capacity $[5,6]$. Different levels of ZEA cause different reactions in animals, and can result in reduced productivity, oxidative damage to organs and tissues, immune stress, reduced reproductive performance, and may even lead to acute death [7-9]. According to recent reports, the toxic effects of ZEA are not limited to estrogen-caused reproductive toxicity, but include other mechanisms that target general cells and tissues $[10,11]$. The oxidative stress caused by the accumulation of reactive oxygen species (ROS) and the regulation of antioxidant enzymes may be one of the main mechanisms of in vivo and in vitro ZEA toxicity [12].

It has been reported that sensitivity to ZEA and its metabolites varies in different experimental animals and livestock species. Pigs are most sensitive to ZEA; this is especially the case for prepubescent sows, as this developmental stage is the most susceptible to the influence of ZEA activity $[13,14]$. Some studies have shown that pigs effectively absorb orally administered ZEA, at a rate of up to $85 \%$ [15]. Once ingested, it can be metabolized to form $\alpha$-zearalenol and $\beta$-zearalenol through intestinal tissue and liver circulation [16]. After penetrating the intestinal wall, ZEA enters the bloodstream and spreads throughout the body, which may cause certain diseases [17]. Therefore, the toxic effects of ZEA on the intestinal tract have increasingly received attention from researchers. To date, most of these studies have focused on morphological changes to the intestines (villus height, crypt depth, villus-gland ratio) [18]. Some studies have shown that ZEA is absorbed in the jejunum and ileum and that it causes oxidative stress [19,20]; however, there are few studies on ZEA-induced oxidative stress in the duodenum of weaned piglets.

The Kelch-like erythroid cell-derived protein with CNC homology-associated protein 1-nuclear factor erythroid 2-related factor 2 (Keap1-Nrf2) signaling pathway is a redoxsensitive signal transduction pathway that is a particularly important anti-oxidative-stress mechanism in the digestive system [21]. Studies have confirmed more than two hundred protective genes downstream of the Keap1-Nrf2 signaling pathway, including genes encoding antioxidant enzyme proteins and phase II detoxification enzymes, all of which play an important role in maintaining tissue homeostasis [22]. Therefore, this signaling pathway has become an important area of focus in the field of oxidative stress research. Oxidative damage in vivo can activate signaling pathways through the transcription of these cell-protection genes.

Although ZEA has been thoroughly studied, the mechanism by which its oxidative stress-related toxicity is mediated is unknown, as is the ZEA-mediated Keap1-Nrf2 signaling pathway. Although observable changes are negligible under physiological conditions, any disruption to organ and tissue function by ZEA may impair homeostasis and thus affect the healthy growth of the body. The purpose of this study was to explore the mechanism of ZEA-induced duodenal oxidative stress in post-weaning gilts in order to find a new theoretical basis and potential avenues for the treatment and prevention of ZEA-induced intestinal oxidative stress in post-weaning gilts.

\section{MATERIALS AND METHODS}

All animals used in the present study were cared for in accordance with the guidelines for the care and use of laboratory animals set by the Animal Nutrition Research Institute of Shandong Agricultural University (Taian, Shandong, China) and the Ministry of Agriculture of China (Beijing, China).

\section{Preparation of zearalenone-zontaminated diets}

Purified ZEA (Fermentek Ltd., Jerusalem, Israel) was first dissolved in acetic ether, and then poured onto talcum powder. The ZEA premix was prepared by blending ZEAcontaminated talcum powder with ZEA-free corn, which was subsequently mixed with a corn-soybean meal to prepare ZEA-contaminated diets 0 (Control), 0.5 (ZEA0.5), 1.0 (ZEA1.0), and 1.5 (ZEA1.5) mg/kg. We chose the concentration range of 0.5 to $1.5 \mathrm{mg} \mathrm{ZEA} / \mathrm{kg}$ considering the study of Jiang et al $[23,24]$ and the feeding situation in China. The maximum allowable ZEA concentration in the diet of postweaning gilts is $0.5 \mathrm{mg} / \mathrm{kg}$ in Chinese Feeding Standard. However, swine diets occasionally contain 1 to $1.5 \mathrm{mg} / \mathrm{kg}$ ZEA [4]. In the present study, all diets were prepared in one batch, and then stored in covered containers before feeding. A composite sample of each experimental diet was prepared to determine the concentration of ZEA and other mycotoxins at the start and end of the feeding experiment. Zearalenone was analyzed by immunoaffinity column chromatography. Aflatoxin (AFL) was measured by liquid chromatographyfluorescence detection, while deoxynivalenol (DON) was measured by liquid chromatography combined with UV detection. The detection limit was $1.0 \mu \mathrm{g} / \mathrm{kg}, 0.1 \mathrm{mg} / \mathrm{kg}$, and $0.1 \mathrm{mg} / \mathrm{kg}$ for AFL, ZEA, and DON, respectively (sum of 3 -acetyl DON, 15-acetyl DON, and nivalenol). The ZEA concentration was $<0.1,0.52 \pm 0.07,1.04 \pm 0.03$, and $1.51 \pm$ $0.13 \mathrm{mg} / \mathrm{kg}$ in the control, ZEA0.5, ZEA1.0, and ZEA1.5 respectively. Aflatoxin and DON were not detected in any of the diets.

\section{Experimental design, animals, and management} Forty post-weaning gilts (Landrace $\times$ Yorkshire $\times$ Duroc) with an average body weight of $14.01 \pm 0.86 \mathrm{~kg}$ were randomly allocated to four experimental groups after $10 \mathrm{~d}$ of acclimation. 
The four groups of piglets were then randomly assigned to the four experimental diets as described above and fed the respective diet for $35 \mathrm{~d}$. The treatments were arranged as a completely randomized design. All piglets were individually housed in cages in a temperature-controlled room at the Animal Husbandry Science and Technology Park of Shandong Agricultural University. The diets (Table 1) used in the study were isocaloric and isonitrogenous, with the only difference being the ZEA concentration. The concentration of all nutrients met or exceeded the minimum requirements established by the NRC [26]. Representative samples of each diet were collected at the beginning and end of the experiment for nutrient analyses using the AOAC methods [27].

\section{Sample collection and preparation}

On the last day of the feeding experiment, the piglets were euthanized after fasting for $12 \mathrm{~h}$. The duodenum was immediately isolated from the body, and then dissected into four portions. Three portions were immediately frozen in liquid nitrogen and stored at $-80^{\circ} \mathrm{C}$ for the subsequent analysis of antioxidant enzyme activity, gene expression, and western blotting. The remaining portion was quickly fixed in Bouin's solution for 24 to $48 \mathrm{~h}$ using graded alcohol concentrations for hematoxylin and eosin staining and immunohistochemical analysis.

Table 1. Ingredients and nutrient levels of the basal diet (air dry basis, $\%)$

\begin{tabular}{|c|c|}
\hline Items & Composition \\
\hline \multicolumn{2}{|l|}{ Ingredients (\%) } \\
\hline Corn & 64.5 \\
\hline Whey powder & 5.0 \\
\hline Soybean meal & 23.0 \\
\hline Fish meal & 5.0 \\
\hline L-lysine $\mathrm{HCl}$ & 0.2 \\
\hline $\mathrm{CaHPO}_{4}$ & 0.7 \\
\hline Pulverized limestone & 0.3 \\
\hline $\mathrm{NaCl}$ & 0.3 \\
\hline Premix $^{1)}$ & 1.0 \\
\hline \multicolumn{2}{|l|}{ Nutrients ${ }^{2)}$} \\
\hline Digestible energy (MJ/kg) & 13.81 \\
\hline Crude protein (\%) & 19.82 \\
\hline Calcium (\%) & 0.70 \\
\hline Total phosphorus (\%) & 0.64 \\
\hline Lysine (\%) & 1.22 \\
\hline Sulfur amino acid (\%) & 0.65 \\
\hline Threonine (\%) & 0.75 \\
\hline Trptophan (\%) & 0.22 \\
\hline \multicolumn{2}{|c|}{$\begin{array}{l}\text { 1) Premix provided the following per kilogram of diet: 3,300 IU vitamin } A \text {, } \\
330 \mathrm{IU} \text { vitamin } D_{3}, 24 \mathrm{IU} \text { vitamin } \mathrm{E}, 0.75 \mathrm{mg} \text { vitamin } \mathrm{K}_{3}, 1.50 \mathrm{mg} \text { vitamin } \\
\mathrm{B}_{1}, 5.25 \mathrm{mg} \text { vitamin } \mathrm{B}_{2}, 0.02625 \mathrm{mg} \text { vitamin } \mathrm{B}_{12}, 15.00 \mathrm{mg} \text { pantothenate, } \\
22.50 \mathrm{mg} \text { niacin, } 0.075 \mathrm{mg} \text { biotin, } 0.45 \mathrm{mg} \text { folic acid, } 6.00 \mathrm{mg} \mathrm{Mn}, 150 \\
\mathrm{mg} \mathrm{Fe}, 150 \mathrm{mg} \mathrm{Zn}, 9.00 \mathrm{mg} \mathrm{Cu}, 0.21 \mathrm{mg} \mathrm{l} \text {, and } 0.45 \mathrm{mg} \text { Se. } \\
\text { 2) Digestible energy was obtained from digestion experiment [25], where- } \\
\text { as the other nutrient contents were calculated values. }\end{array}$} \\
\hline
\end{tabular}

\section{Determination of antioxidant enzyme activity}

The frozen duodenum samples were thawed, rinsed with ice-cold deionized water, and dried using a filter paper. The samples were then homogenized with $0.02 \mathrm{mmol} / \mathrm{L}$ Tris $\mathrm{HCl}(\mathrm{pH} 7.4)$ at a ratio of $1: 10(\mathrm{mg} / \mathrm{mL})$, followed by centrifugation $(10,000 \times \mathrm{g})$ at $4^{\circ} \mathrm{C}$ for $15 \mathrm{~min}$. The supernatant was collected to analyze the total superoxide dismutase ( $\mathrm{T}$ SOD) and glutathione peroxidase (GSH-PX) activities, and malondialdehyde (MDA) concentration using the methods described by Jiang et al [28] with SOD A001-1, GSH-PX A005, and MDA A003 Assay Kits, respectively (Nanjing Jiancheng Bioengineering Institute, Nanjing, China). The protein concentration was also determined using the method of Bradford [29] with a protein assay kit (A045; Nanjing Jiancheng Bioengineering Institute, China).

\section{Immunohistochemical analysis for integrated optical density of Nrf2 and GPX1}

For this analysis, the duodenum samples stored in Bouin's solution were sliced into $5-\mu \mathrm{m}$ sections using a Leica microtome (RM 2235; Leica Biosystems Nussloch GmbH, Nussloch, Germany). The sections were then processed by immobilization on poly-l-lysine-coated glass slides, drying overnight at $37^{\circ} \mathrm{C}$, dewaxing, rehydration, and antigen retrieval in sodium citrate buffer $(0.01 \mathrm{~mol} / \mathrm{L}, \mathrm{pH} 6.0)$ using a microwave unit for $20 \mathrm{~min}$ at full power, which was followed by washing three times ( 5 min per washing) with phosphate buffered solution $(0.01 \mathrm{~mol} / \mathrm{L}, \mathrm{pH} 7.2)$. The subsequent sample processing for immunohistochemical analysis was the same as that described by Zhou et al [30].

To evaluate the extent of staining and quantity of the target antigen in Nrf2- and glutathione peroxidase 1 (GPX1)-positive cells, images were randomly captured using a microscopic camera system at $100 \times$ magnification, which was then analyzed using image analysis software (Image-Pro Plus 6.0; Media Cybernetics, Inc., Rockville, MD, USA) to obtain the total cross-sectional integrated optical density (IOD) [31]. Six stained samples randomly selected from 10 piglets per treatment were used in this analysis.

\section{Quantification of Keap1, Nrf2, GPX1, NQO1, HO1, GCLM, GCLC, and GAPDH mRNA expression using quantitative real-time polymerase chain reaction} The total RNA was extracted from the duodenum samples stored at $-80^{\circ} \mathrm{C}$ using RNAiso Plus (D9108B; Applied TaKaRa, DaLian, China) according to the manufacturer's instructions. The purity and concentration of the RNA were assessed using an Eppendorf Biophotometer (RS323C; Eppendorf Aktien Gesellschaft, Hamburg, Germany) at an absorbance ratio of 260:280 nm (a range of 1.8 to 2.0 indicates pure RNA sample). The RNA integrity was verified by agarose gel electrophoresis. The total RNA was reverse transcribed 
to cDNA using a Reverse Transcription System Kit (PrimeScript RT Master Mix, RR036A; Applied TaKaRa, China).

The polymerase chain reaction (PCR) mixture of total volume $20 \mu \mathrm{L}$, containing $10 \mu \mathrm{L}$ of SYBRY Premix Ex Taq II, $0.4 \mu \mathrm{L}$ of DyeII (SYBRY Premix Ex Taq-TIi RNaseH Plus, DRR420A; Applied TaKaRa, China), $0.4 \mu \mathrm{L}$ of both forward and reverse primers, and $2 \mu \mathrm{L}$ of cDNA ( $<100 \mathrm{ng})$, was used for the quantitative real-time PCR (qRT-PCR) analysis. The optimized qRT-PCR protocol included an initial denaturation step at $95^{\circ} \mathrm{C}$ for $30 \mathrm{~s}$, followed by 43 cycles at $95^{\circ} \mathrm{C}$ for $5 \mathrm{~s}, 60^{\circ} \mathrm{C}$ for $34 \mathrm{~s}, 95^{\circ} \mathrm{C}$ for $15 \mathrm{~s}, 60^{\circ} \mathrm{C}$ for $60 \mathrm{~s}$, and $95^{\circ} \mathrm{C}$ for $15 \mathrm{~s}$. The qRT-PCRs were conducted in an AB 7500 Real Time PCR System (Applied Biosystems, Foster City, CA, USA). The relative expression level of Keap1, Nrf2, GPX1, quinone oxidoreductase 1 (NQO1), hemeoxygenase 1 (HO1), modifier subunit of glutamate-cysteine ligase (GCLM), catalytic subunit of glutamate-cysteine ligase (GCLC), and glyceraldehyde-3-phosphate dehydrogenase mRNA was calculated using the $2^{-\Delta \Delta \mathrm{Ct}}$ method [32]. The analysis was repeated thrice per sample. The sequence and production length of primers are presented in Table 2.

\section{Nuclear protein extraction and western blot analysis}

The total protein was extracted from the duodenum samples stored at $-80^{\circ} \mathrm{C}$ using radioimmunoprecipitation assay lysis buffer supplemented with phenylmethanesulfonyl fluoride (Beyotime Biotechnology, Shanghai, China) according to the manufacturer's instructions. The samples were incubated on ice for $30 \mathrm{~min}$ and the supernatant was collected by centrifugation $(12,500 \times \mathrm{g})$ at $4^{\circ} \mathrm{C}$ for $10 \mathrm{~min}$. After protein quantification using the Bicinchoninic Acid Protein Assay Kit (Tiangen Biotech Co., Ltd., Beijing, China), the solution containing $50 \mu \mathrm{g}$ of total protein was loaded onto a sodium dodecyl sulfate polyacrylamide gel and subjected to electrophoresis. The proteins were transferred on to $0.22-\mu \mathrm{m}$ polyvinylidene difluoride (PVDF) membranes (Solarbio, Beijing, China). After blocking the PVDF membranes in 5\% skim milk for $2 \mathrm{~h}$, the blots were washed for $30 \mathrm{~min}$ with Tris-buffered saline containing $0.1 \%$ Tween 20 (TBST: 20 mM Tris, pH 7.5; 150 $\mathrm{mM} \mathrm{NaCl}$; and $0.1 \%$ Tween-20) and incubated overnight at $4^{\circ} \mathrm{C}$ with the following primary antibodies: Nrf2 (1:1,000; sc-722, Santa Cruz Biotechnology, Shanghai, China), GPX1 (1:1,000; sc-22145, Santa Cruz Biotechnology, China), Keap1 (1:500; ab218815, Abcam, Shanghai, China), HO1 (1:10,000; ab68477, Abcam, China), NQO1 (1:10,000; ab80588, Abcam, China), GCLM (1:5,000; ab124827, Abcam, China), GCLC (1:10,000; ab190685, Abcam, China), and $\beta$-actin (1:2,000; Beyotime Biotechnology, China). The membranes were then washed in TBST for $30 \mathrm{~min}$ and incubated again at $37^{\circ} \mathrm{C}$ with anti-rabbit immunoglobulin $\mathrm{G}$ (IgG) antibody (1:5,000; Beyotime Biotechnology, China), and anti-mouse IgG (1:5,000; Beyotime Biotechnology, China) for $1.5 \mathrm{~h}$. Following washing with TBST for $30 \mathrm{~min}$, the membranes were immersed in a high-sensitivity luminescence reagent (BeyoECL Plus; Beyotime Biotechnology, China), and then exposed to film using a Fusion FX imaging system and analyzed using a FusionCapt Advance FX7 software (Beijing Oriental Science and Technology Development Co., Ltd., Beijing, China). The concentration of proteins was determined using Image-Pro Plus 6.0 (Media Cybernetics, Inc., USA).

Table 2. Sequence of primers for real-time polymerase chain reaction

\begin{tabular}{|c|c|c|c|}
\hline Target gene & Accession No. & Primer Sequence $\left(5^{\prime} \text { to } 3^{\prime}\right)^{1)}$ & Product size (bp) \\
\hline \multirow[t]{2}{*}{ Nrf2 } & RA_011763 & F: GAAAGCCCAGTCTTCATTGC & 190 \\
\hline & & R: TTGGAACCGTGCTAGTCTCA & \\
\hline GPX1 & & R: AGGCGAAGAGCGGGTGAGCA & \\
\hline \multirow[t]{2}{*}{ Keap1 } & NM_001114671.1 & F: GCCTCATCGAGTTCGCTTAC & 105 \\
\hline & & R: CACGGACCACACTGTCAATC & \\
\hline HO1 & & R: TGTAGACCGGGTTCTCCTTG & \\
\hline \multirow[t]{2}{*}{ NQ01 } & NM_ 001159613.1 & F: TGAATTACATCTCTGTGGTTTA & 171 \\
\hline & & R: AGAATGACACTCATATTAGGCG & \\
\hline \multirow[t]{2}{*}{ GCLM } & CV868255.1 & F: ACAATACAACGGTTCAGGTGAGT & 122 \\
\hline & & R: GCCTGTAAAATGTGTCATTGAGG & \\
\hline
\end{tabular}

Nrf2, nuclear factor erythroid 2-related factor 2; Keap1, Kelch-like ECH-associated protein1; GPX1, glutathione peroxidase 1; H01, hemeoxygenase 1; NQ01, quinone oxidoreductase 1; GCLM, modifier subunit of glutamate-cysteine ligase; GCLC, catalytic subunit of glutamate-cysteine ligase; GAPDH, glyceraldehyde-3-phosphate dehydrogenase.

1) $F$, forward primer; $R$, reverse primer. 


\section{Statistical analysis}

All data were subjected to one-way analysis of variance using the generalized linear model procedure of SAS 9.2 (SAS Inst. Inc., Cary, NC, USA). The data were initially analyzed as a completely randomized design, with treatment as the fixed effect and individual piglet as random factor. Orthogonal polynomial contrasts were used to determine linear and quadratic responses to the dietary ZEA concentrations. The significance of differences among treatments was tested using Duncan's multiple range tests and significance was declared at $\mathrm{p}<0.05$.

\section{RESULTS}

Effects of ZEA on antioxidant enzyme activity of the duodenum

The activities of T-SOD and GSH-Px in the duodenum decreased linearly $(\mathrm{p}<0.05)$ and quadratically $(\mathrm{p}<0.05)$ as the dietary ZEA concentration increased from 0 to $1.5 \mathrm{mg} / \mathrm{kg}$ (Table 3). The MDA concentration in the duodenum was significant increased $(\mathrm{p}<0.05)$ in the ZEA1.0 and ZEA1.5 group. The lowest $(\mathrm{p}<0.05)$ T-SOD and GSH-Px activities were observed in piglets in the ZEA1.5 group, and the highest MDA concentration $(\mathrm{p}<0.05)$ was observed the ZEA1.0 group.

\section{Effects of ZEA on Nrf2 and GPX1 immunoreactivities} in the duodenum

Immunohistochemical analysis showed that the Nrf2 and GPX1 immunoreactive substances were mainly localized in the lamina propria (S) around the intestine gland $(G)$, whereas negative or faint Nrf2 and GPX1 immunoreactivity was observed in most of the intestinal villus epithelium of the duodenum (Nrf2, Figure 1; GPX1, Figure 2). Staining revealed a light yellow immunoreactive substance indicating Nrf2 and GPX1 in the control (A). The localization pattern of positive substances found in the ZEA-treated piglets was comparable with that in the control (red arrows). However, the positive reactions of Nrf2 and GPX1 were linearly $(\mathrm{p}<$ $0.05)$ and quadratically $(\mathrm{p}<0.05)$ enhanced (A1-B1-C1-D1) compared with those of the control by increased ZEA concentrations (Table 4), as indicated by the IOD values.
Effects of ZEA on the relative mRNA expressions of Keap1, Nrf2, GPX1, NQO1, HO1, GCLM, and GCLC The relative duodenal mRNA expressions of Nrf2 and GPX1 increased both linearly and quadratically $(\mathrm{p}<0.05)$ in each of the treatment groups (Table 5). However, the relative mRNA expressions of Keap 1 and GCLC decreased $(\mathrm{p}<0.05)$ linearly and quadratically with the increase in ZEA concentration. Compared to the control group, piglets in each of the treatment groups presented low $(\mathrm{p}<0.05)$ relative mRNA Keap1 expression and high $(\mathrm{p}<0.05)$ relative Nrf2 and GPX1 expressions; the relative mRNA expressions of NQO1 and HO1 were high $(\mathrm{p}<0.05)$ at ZEA concentrations of $0.5 \mathrm{mg} / \mathrm{kg}$ and $1.0 \mathrm{mg} / \mathrm{kg}$, respectively.

\section{Effects of ZEA on the relative expression of Keap1,} Nrf2, GPX1, NQO1, HO1, GCLM, and GCLC proteins Western blot analysis revealed positive bands of appropriate sizes for all the studied proteins (Keap1, Nrf2, GPX1, NQO1, HO1, GCLM, GCLC, and $\beta$-actin; Figure 3). The relative abundances of Nrf2, GPX1, and NQO1 increased $(\mathrm{p}<0.05)$ linearly and quadratically with the increase in ZEA levels. On the other hand, the relative abundances of GCLM decreased $(p<0.05)$ linearly and quadratically, and the relative abundance of Keap1 decreased $(\mathrm{p}<0.05)$ quadratically with increasing ZEA levels. While the expression of most individual proteins varied, but not significantly, over the different dietary ZEA concentrations, the abundance of Keap 1 and GCLM decreased $(\mathrm{p}<0.05)$ and those of Nrf2, GPX1, and NQO1 increased $(p<0.05)$ in all treatment groups. However, the relative abundance of $\mathrm{HO} 1$ decreased $(\mathrm{p}<0.05)$ only in the ZEA1.5 group, and the relative abundance of GCLC increased $(\mathrm{p}<0.05)$ in the ZEA0.5 group and decreased $(\mathrm{p}<0.05)$ in the ZEA1.5 group of piglets.

\section{DISCUSSION}

A growing number of studies show that ZEA can interfere with the antioxidant-related mechanisms of cells; affect the levels and activities of MDA, SOD, GSH-Px, and other antioxidant enzymes in different cell types; and induce oxidative stress [33,34]. In this study, we hypothesized that ZEA induces

Table 3. Effects of zearalenone on antioxidant capacity in the duodenum of post-weaning gilts

\begin{tabular}{|c|c|c|c|c|c|c|c|c|}
\hline \multirow{2}{*}{ Items } & \multirow{2}{*}{ Control $^{1)}$} & \multirow{2}{*}{ ZEA0. $5^{1)}$} & \multirow{2}{*}{ ZEA1.01) } & \multirow{2}{*}{ ZEA1. $5^{1)}$} & \multirow{2}{*}{ SEM } & \multicolumn{3}{|c|}{ p-values } \\
\hline & & & & & & Treatment & Linear & Quadratic \\
\hline T-SOD (U/mg prot) & $19.30^{\mathrm{a}}$ & $13.14^{b}$ & $12.05^{c}$ & $11.37^{d}$ & 0.109 & $<0.001$ & $<0.001$ & $<0.001$ \\
\hline GSH-Px (U/mL) & $165.89^{\mathrm{a}}$ & $136.53^{b}$ & $122.73^{c}$ & $112.29^{d}$ & 0.845 & $<0.001$ & $<0.001$ & $<0.001$ \\
\hline MDA (nmol/mg prot) & $4.62^{c}$ & $4.55^{c}$ & $6.54^{\mathrm{a}}$ & $4.99^{b}$ & 0.048 & $<0.001$ & 0.106 & 0.045 \\
\hline
\end{tabular}

SEM, standard error of the mean; T-SOD, total superoxide dismutase; GSH-PX, glutathione peroxidase; MDA, malondialdehyde.

1) Control, ZEA0.5, ZEA1.0, and ZEA1.5 represent the control diet with an addition of $0,0.5,1.0$, and $1.5 \mathrm{mg} / \mathrm{kg}$ ZEA, and with analyzed ZEA concentrations of $<0.1,0.52 \pm 0.07,1.04 \pm 0.03$, and $1.51 \pm 0.13 \mathrm{mg} / \mathrm{kg}$, respectively.

a-d $V$ alues within a row with the different letters mean significantly different $(p<0.05)$. 

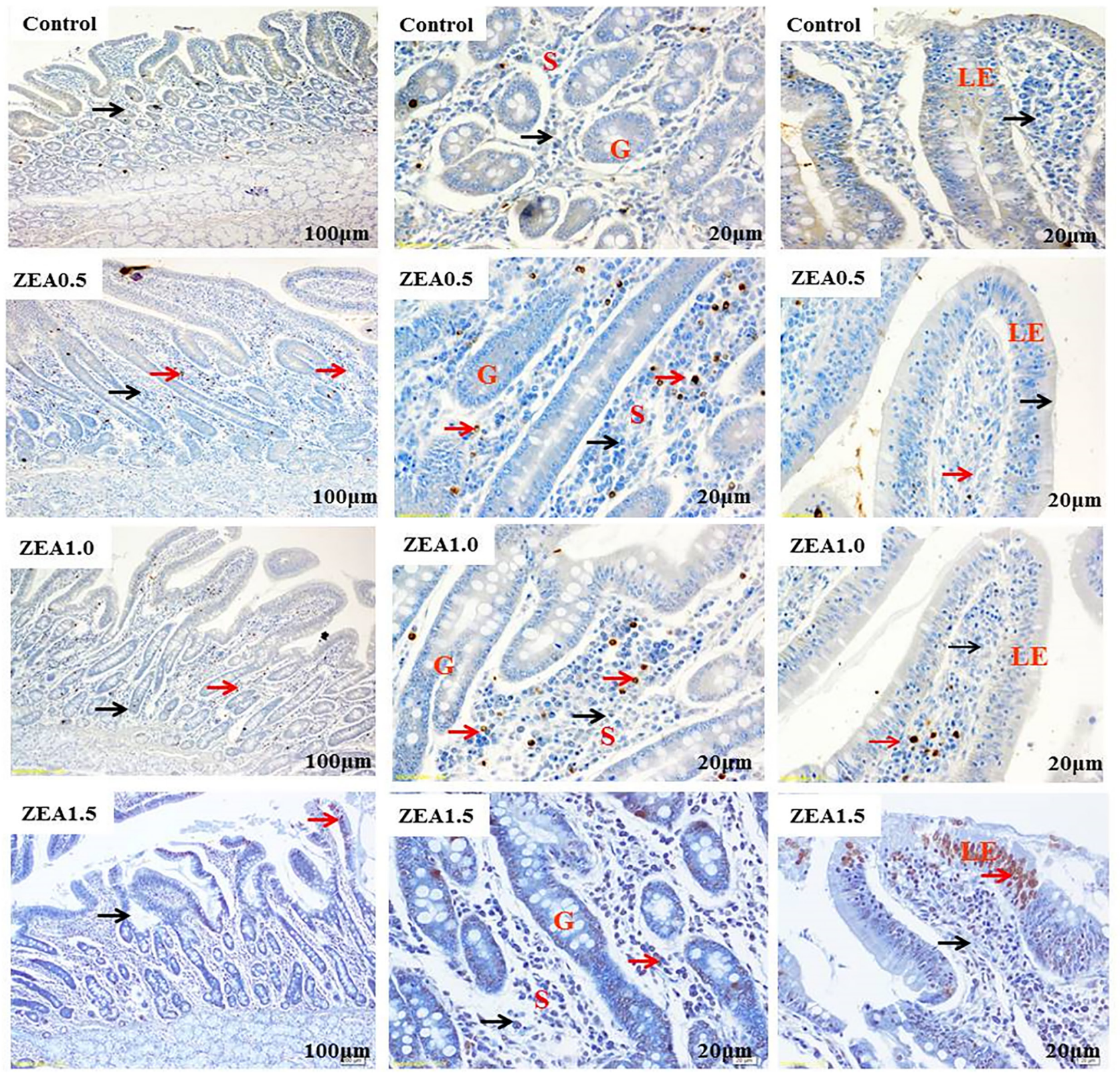

Figure 1. Effects of zearalenone (ZEA) on the localization of nuclear factor erythroid 2-related factor 2 (Nrf2) in the duodenum of post-weaning gilts. All four treatments are shown as follows: Control (A1, A2, and A3); ZEA0.5 treatment (B1, B2, and B3); ZEA1.0 treatment (C1, C2, and C3); and ZEA1.5 treatment (D1, D2, and D3). Control, ZEA0.5, ZEA1.0, and ZEA1.5 represent the control diet with an addition of 0, 0.5, 1.0, and 1.5 mg/kg ZEA, and with analyzed ZEA concentrations of $<0.1,0.52 \pm 0.07,1.04 \pm 0.03$, and $1.51 \pm 0.13 \mathrm{mg} / \mathrm{kg}$, respectively. LE is intestinal villus epithelium, $\mathrm{G}$ is intestinal gland, and $\mathrm{S}$ is lamina propria. Red arrows represent immunoreactive cells of Nrf2 and blue arrows represent immune-negative cells of Nrf2. Scale bars: approximately $20 \mu \mathrm{m}$ for A2, B2, C2, D2, A3, B3, C3, D3, and $100 \mu \mathrm{m}$ for A1, B1, C1, D1.

oxidative stress in the duodenum of post-weaning gilts. In order to demonstrate this, we evaluated the level of MDA (a product of lipid peroxidation of ROS in duodenal cells), along with changes in the activities of T-SOD and GSH-Px as three indicators of oxidative stress. The experimental data support our conjecture by showing that ZEA significantly reduces the activities of the antioxidant enzymes T-SOD and GSH-Px, increases the MDA content, and causes oxidative stress in the duodenum. Much previous research on the oxidative stress caused by ZEA has revealed that different concentrations of ZEA can significantly increase the MDA levels of human hepatoma cells [35] and Caco-2 cells [36], thus causing oxidative stress. Ren et al $[37,38]$ found that ZEA can reduce the antioxidant enzyme activity of the spleen in mice and increase MDA content. It has been found that dietary contamination with ZEA $(1.0 \mathrm{mg} / \mathrm{kg})$ can significantly reduce SOD and GSH-Px activities and significantly increase MDA content in the livers of weaned piglets [39]. 

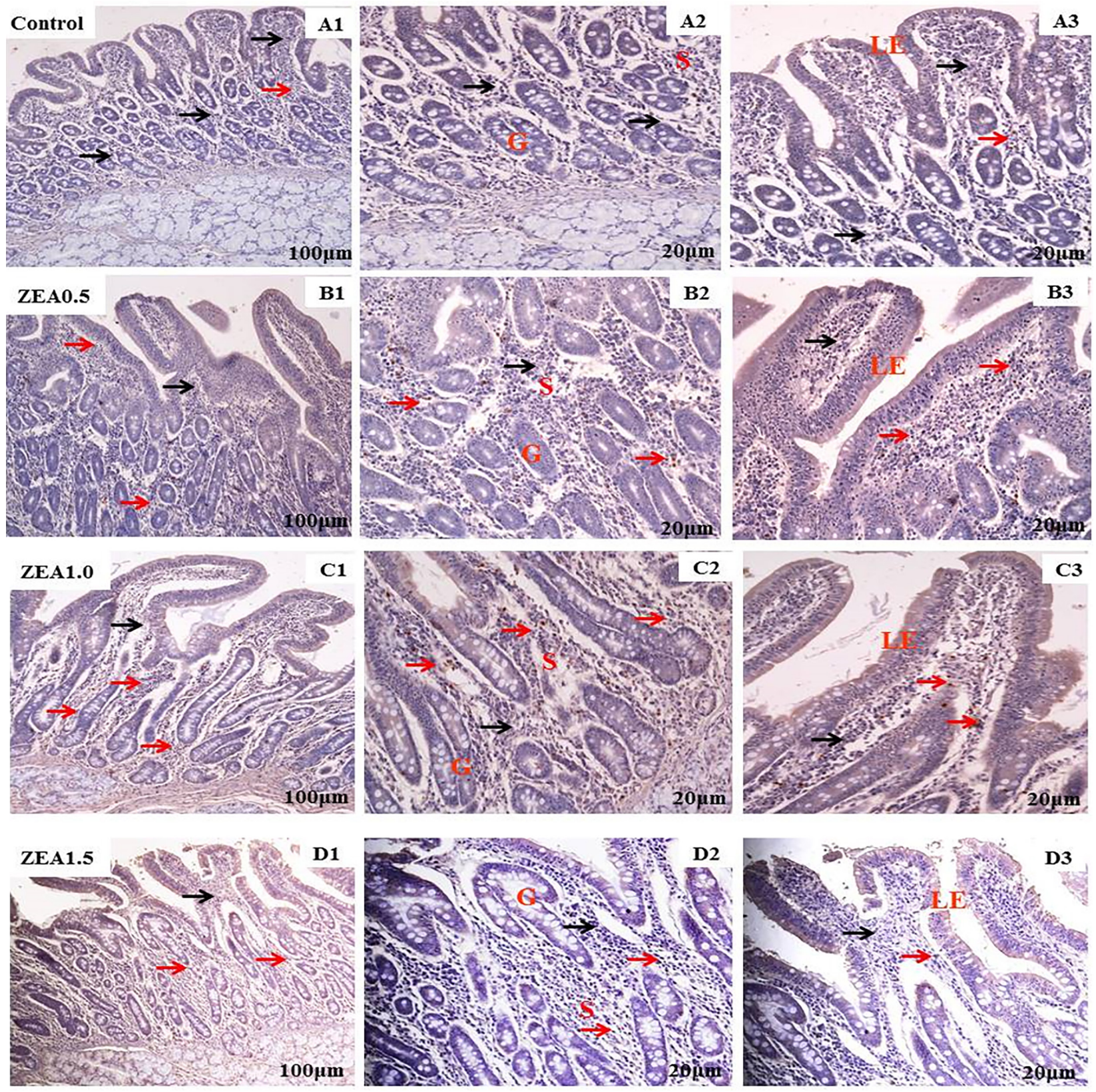

Figure 2. Effects of zearalenone (ZEA) on the localization of glutathione peroxidase 1 (GPX1) in the duodenum of post-weaning gilts. All four treatments are shown as follows: Control (A1, A2, and A3); ZEA0.5 treatment (B1, B2, and B3); ZEA1.0 treatment (C1, C2, and C3); and ZEA1.5 treatment (D1, D2, and D3). Control, ZEA0.5, ZEA1.0, and ZEA1.5 represent the control diet with an addition of 0, 0.5, 1.0, and 1.5 mg/kg ZEA, and with analyzed ZEA concentrations of $<0.1,0.52 \pm 0.07,1.04 \pm 0.03$, and $1.51 \pm 0.13 \mathrm{mg} / \mathrm{kg}$, respectively. LE is intestinal villus epithelium, $\mathrm{G}$ is intestinal gland, and $\mathrm{S}$ is lamina propria. Red arrows represent immunoreactive cells of GPX1 and blue arrows represent immune-negative cells of GPX1. Scale bars: approximately $20 \mu \mathrm{m}$ for A2, B2, C2, D2, A3, B3, C3, D3, and $100 \mu \mathrm{m}$ for A1, B1, C1, D1.

Table 4. Effects of zearalenone on the immunoreactive intergrated optic density of Nrf2 and GPX1 in the duodenum of post-weaning gilts ( $\left.\times 10^{3}\right)$

\begin{tabular}{|c|c|c|c|c|c|c|c|c|}
\hline \multirow{2}{*}{ Items } & \multirow{2}{*}{ Control $^{1)}$} & \multirow{2}{*}{ ZEA0. $5^{1)}$} & \multirow{2}{*}{ ZEA1.01) } & \multirow{2}{*}{ ZEA1.5 ${ }^{1)}$} & \multirow{2}{*}{ SEM } & \multicolumn{3}{|c|}{ p-values } \\
\hline & & & & & & Treatment & Linear & Quadratic \\
\hline Nrf2 & $0.46^{b}$ & $0.97^{a}$ & $0.92^{a}$ & $0.97^{a}$ & 0.019 & $<0.001$ & $<0.001$ & $<0.001$ \\
\hline
\end{tabular}

SEM, standard error of the mean; Nrf2, nuclear factor erythroid 2-related factor 2; GPX1, glutathione peroxidase 1.

1) Control, ZEA0.5, ZEA1.0, and ZEA1.5 represent the control diet with an addition of 0, 0.5, 1.0, and $1.5 \mathrm{mg} / \mathrm{kg}$ ZEA, and with analyzed ZEA concentrations of $<0.1,0.52 \pm 0.07,1.04 \pm 0.03$, and $1.51 \pm 0.13 \mathrm{mg} / \mathrm{kg}$, respectively.

a,b $V$ alues within a row with the different letters mean significantly different $(p<0.05)$. 
Table 5. Effects of zearalenone on the relative mRNA expressions of Keap1, Nrf2, GPX1, NQ01, H01, GCLM, and GCLC in the duodenum of post-weaning gilts

\begin{tabular}{|c|c|c|c|c|c|c|c|c|}
\hline \multirow{2}{*}{ Items } & \multirow{2}{*}{ Control $^{1)}$} & \multirow{2}{*}{ ZEA0. $5^{1)}$} & \multirow{2}{*}{ ZEA1.01) } & \multirow{2}{*}{ ZEA1. $5^{1)}$} & \multirow{2}{*}{ SEM } & \multicolumn{3}{|c|}{ p-values } \\
\hline & & & & & & Treatment & Linear & Quadratic \\
\hline Keap1 & $1.01^{a}$ & $1.10^{a}$ & $0.63^{b}$ & $0.26^{c}$ & 0.018 & $<0.001$ & 0.022 & 0.005 \\
\hline GPX1 & $1.08^{\mathrm{c}}$ & $1.21^{b}$ & $1.39^{a}$ & $1.39^{\mathrm{a}}$ & 0.014 & $<0.001$ & $<0.001$ & $<0.001$ \\
\hline NQ01 & $1.00^{b}$ & $1.16^{a}$ & $0.25^{c}$ & $0.19^{c}$ & 0.022 & $<0.001$ & $<0.001$ & $<0.001$ \\
\hline HO1 & $1.00^{b}$ & $0.44^{c}$ & $1.67^{\mathrm{a}}$ & $0.26^{c}$ & 0.034 & $<0.001$ & 0.349 & 0.128 \\
\hline
\end{tabular}

SEM, standard error of the mean; Keap1, Kelch-like ECH-associated protein1; Nrf2, nuclear factor erythroid 2-related factor 2; GPX1, glutathione peroxidase 1 ; NQ01, quinone oxidoreductase 1; H01, hemeoxygenase 1; GCLM, modifier subunit of glutamate-cysteine ligase; GCLC, catalytic subunit of glutamate-cysteine ligase.

1) Control, ZEA0.5, ZEA1.0, and ZEA1.5 represent the control diet with an addition of $0,0.5,1.0$, and $1.5 \mathrm{mg} / \mathrm{kg}$ ZEA, and with analyzed ZEA concentrations of $<0.1,0.52 \pm 0.07,1.04 \pm 0.03$, and $1.51 \pm 0.13 \mathrm{mg} / \mathrm{kg}$, respectively.

${ }^{a-c}$ Values within a row with the different letters mean significantly different $(p<0.05)$.

Our previous studies have shown that ZEA can significantly reduce T-SOD and GSH-Px activities and increase MDA content in the jejunum and ileum, which further supports our conjecture that ZEA can induce intestinal oxidative stress in weaned piglets $[20,40]$. More significantly, we found increased GPX1 immunoreactivity in the lamina propria of the duodenal gland of post-weaning gilts fed a ZEA-contaminated diet; this was confirmed by the deep staining that indicated the presence of this protein. This finding is consistent with the significant increase in relative mRNA and protein expressions of the antioxidant gene GPX1. We believe that the increase in GPX1 expression may be a protective mechanism by which ZEA-induced oxidative stress is resisted in the duodenum of post-weaning gilts. In order to better study the mechanism of ZEA-induced oxidative stress, we conducted a more in-depth experiment.

In recent years, the Keap1-Nrf2 signaling pathway has become recognized as one of the most important mechanisms of cellular antioxidant defense, and has therefore been the focus of much research [41,42]. A key transcription factor of this signaling pathway that is responsive to anti-oxidative stress is Nrf2, which is highly expressed in the gastrointestinal tract [21]. Activated Nrf2 can bind with the antioxidant response element (ARE) to form the Nrf2/ARE signal transduction pathway and induce the transcription of downstream antioxidant genes (e.g., GPX1) and the phase II detoxification enzyme genes HO1, NQO1, GCLM, and GCLC [43]. Under normal physiological conditions, Keap1 combines with $\mathrm{Nrf2}$ in the cytoplasm to form a dimer. In this way, Keap1 negatively regulates $\mathrm{Nrf2}$, inhibiting its activity and thereby maintaining cell homeostasis. When exposed to poisons or certain stimuli, the Keap1-Nrf2 dimer is automatically separated, and Nrf2 is activated and transferred to the nucleus to bind to ARE, thereby activating the expression of antioxidant enzymes and phase II detoxification enzyme genes
$[44,45]$. Therefore, the decrease of antioxidant enzyme activity and the increase of GPX1 expression in intestinal oxidative stress induced by ZEA may be the result of an oxidative stress signal induced by the Keap1-Nrf2 signaling pathway and the regulation of antioxidant enzyme expression by the transcription factor Nrf2.

In this study, we found enhanced Nrf2 immunoreactivity in the duodenal gland of post-weaning gilts fed a ZEA-contaminated diet. Deepened staining indicated a positive reaction, and significant increases were observed in the relative mRNA expression and protein level of Nrf2 in the duodenum. These results indicate that $\mathrm{Nrf} 2$ is activated under oxidative stress and may protect the duodenum from the ensuing effects. We further found that the relative mRNA and protein expression of Keap1 decreased significantly, and the relative protein abundance of NQO1 increased significantly in the ZEA experimental groups. Interestingly, while Keap1 was significantly reduced at both the mRNA and protein levels in the duodenum, the relative mRNA and protein expression of NQO1, HO1, and GCLM were significantly increased in the jejunum and ileum with ZEAinduced oxidative stress $[20,40]$. These results suggest that ZEA induces more obvious oxidative stress in the jejunum and ileum Moreover, the expression of Nrf2 in the Keap1Nrf2 signaling pathway also increased, confirmed by increases in the optical density of $\mathrm{Nrf2}$ immunoreactive substances. These differences may be due to the different digestion and absorption capacity of ZEA in the duodenum, jejunum, and ileum or to different tolerance of ZEA in the duodenum, jejunum, and ileum. Most importantly, however, when postweaning gilts are fed ZEA-contaminated diets, the Keap1Nrf2 signaling pathway is activated and may play an important role in promoting the health of the duodenum, jejunum, and ileum $[20,40]$. These findings further indicate that the Keap1-Nrf2 signaling pathway in the duodenum of post- 

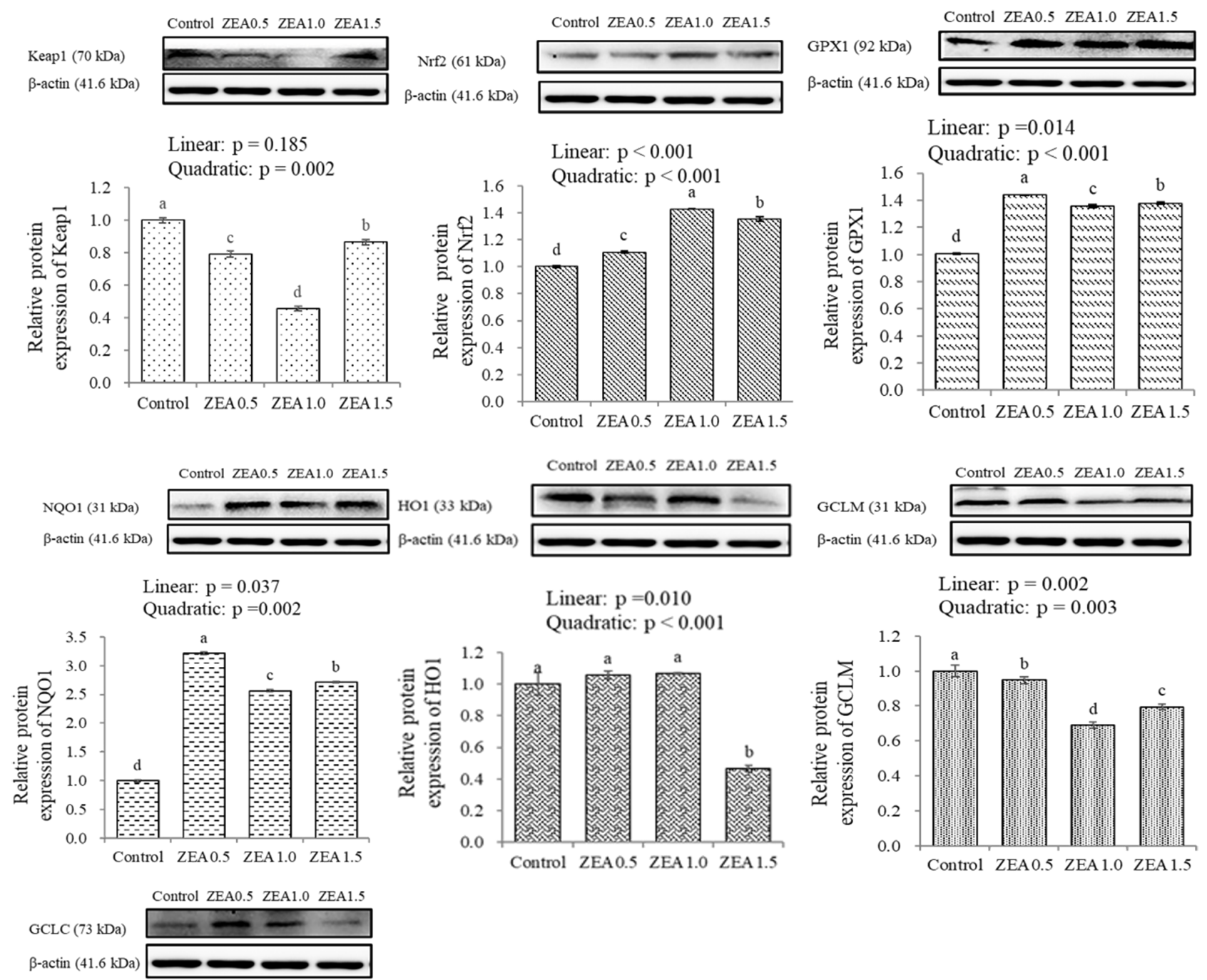

Linear: $\mathrm{p}=0.069$

Quadratic: $\mathrm{p}<0.001$

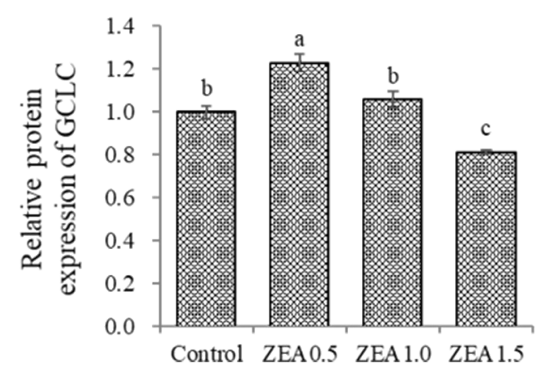

Figure 3. Effects of zearalenone on the protein expressions of Keap1, Nrf2, GPX1, NQ01, H01, GCLM, and GCLC in the duodenum of post-weaning gilts. Control, ZEA0.5, ZEA1.0, and ZEA1.5 represent the control diet with an addition of 0, 0.5, 1.0, and 1.5 mg/kg ZEA, and with analyzed ZEA concentrations of $<0.1,0.52 \pm 0.07,1.04 \pm 0.03$, and $1.51 \pm 0.13 \mathrm{mg} / \mathrm{kg}$, respectively. Nrf2, nuclear factor erythroid 2-related factor 2; Keap1, Kelch-like $\mathrm{ECH}$-associated protein1; GPX1, glutathione peroxidase 1; NQ01, quinone oxidoreductase 1; H01, hemeoxygenase 1; GCLM, modifier subunit of glutamate-cysteine ligase; GCLC, catalytic subunit of glutamate-cysteine ligase. ${ }^{\text {a-d }}$ Different letters indicate significant differences between values $(p<0.05)$.

weaning gilts fed ZEA-contaminated diets is activated and may play an important protective role. Studies have found that increased levels of $\mathrm{Nrf2}$ in mouse hearts and upregulation of antioxidant enzymes regulated by the Keap1-Nrf2 signaling pathway can offset isoproterenol-induced oxidative stress [46]. It has been reported that the levels of HO1, NQO1, and Nrf2 significantly increase in the kidneys of rats subjected to lipopolysaccharide-stimulated oxidative 
stress [47]. The same study found that stimulating the production of $\mathrm{HO} 1$ and $\mathrm{Nrf2}$ proteins can reduce oxidative damage to the livers of mice and regulate the activity of antioxidant enzymes [48]. Similar to the results of this study, the previous studies mentioned further show that the Keap1Nrf2 signaling pathway is activated in response to oxidative stress, and protects the body by resisting it. This shows that ZEA can induce intestinal oxidative stress, and the transcription factor Nrf2 in the Keap1-Nrf2 signaling pathway can be used as a potential therapeutic target to resist the oxidative damage caused by ZEA. Due to the complex in vivo environment of post-weaning gilts, determining the role of the Keap1-Nrf2 signaling pathway in ZEA-induced duodenal oxidative stress requires verification through further experiments.

In summary, our study shows that ZEA reduces the antioxidant enzyme activity of the duodenum of post-weaning gilts and induces duodenal oxidative stress. The increased expression of Nrf2, GPX1, HO1, NQO1 and the significant decrease of Keap1 expression indicate that the Keap1-Nrf2 signaling pathway is activated and may play an important role under conditions of oxidative stress. The protective effect of the Keap1-Nrf2 signaling pathway in ZEA-induced intestinal oxidative stress in piglets provides new possibilities for solving the toxicity of ZEA in the intestinal and digestive systems and also provides new strategies for the healthy production of animals. In order to better study the role of the Keap1-Nrf2 signaling pathway in ZEA-induced intestinal oxidative stress, in vitro cell testing is the goal of our next study.

\section{CONFLICT OF INTEREST}

We certify that there is no conflict of interest with any financial organization regarding the material discussed in the manuscript.

\section{ACKNOWLEDGMENTS}

This study was funded in part by Major Innovative Projects in Shandong Province of Research and application of environment-friendly feed and the critical technologies for pigs and poultry without antibiotic (2019JZZY020609), the Natural Science Foundation of Shandong Province (Project No. ZR2019MC038), the Agriculture Research System in Shandong Province (Project No. SDAIT-08-05), and the Funds of Shandong "Double Tops".

\section{REFERENCES}

1. De Boevre M, Di Mavungu JD, Landschoot S, et al. Natural occurrence of mycotoxins and their masked forms in food and feed products. World Mycotoxin J 2012;5:207-19. https:// doi.org/10.3920/WMJ2012.1410

2. Chang H, Kim W, Park JH, et al. The occurrence of zearalenone in South Korean feedstuffs between 2009 and 2016. Toxins 2017;9:223. https://doi.org/10.3390/toxins9070223

3. Price WD, Lovell RA, McChesney DG. Naturally occurring toxins in feedstuffs: center for veterinary medicine perspective. J Anim Sci 1993;71:2556-62. https://doi.org/10.2527/ 1993.7192556x

4. Zinedine A, Soriano JM, Molto JC, Manes J. Review on the toxicity, occurrence, metabolism, detoxification, regulations and intake of zearalenone: an oestrogenic mycotoxin. Food Chem Toxicol 2007;45:1-18. https://doi.org/10.1016/j.fct.2006. 07.030

5. Takemura H, Shim JY, Sayama K, Tsubura A, Zhu BT, Shimoi K. Characterization of the estrogenic activities of zearalenone and zeranol in vivo and in vitro. J Steroid Biochem Mol Biol 2007;103:170-7. https://doi.org/10.1016/j.jsbmb.2006.08.008

6. Wielogórska E, Elliott CT, Danaher M, Connolly L. Validation and application of a reporter gene assay for the determination of estrogenic endocrine disruptor activity in milk. Food Chem Toxicol 2014;69:260-6. https://doi.org/10.1016/ j.fct.2014.04.028

7. Dai M, Jiang S, Yuan X, Yang W, Yang Z, Huang L. Effects of zearalenone-diet on expression of ghrelin and PCNA genes in ovaries of post-weaning piglets. Anim Reprod Sci 2016; 168:126-37. https://doi.org/10.1016/j.anireprosci.2016.03. 006

8. Chen XX, Yang CW, Huang LB, Niu QS, Jiang SZ, Chi F. Zearalenone altered the serum hormones, morphologic and apoptotic measurements of genital organs in post-weaning gilts. Asian-Australas J Anim Sci 2015;28:171-9. https://doi. org/10.5713/ajas.14.0329

9. Yang LJ, Zhou M, Huang LB, et al. Zearalenone-promoted follicle growth through modulation of Wnt- $1 / \beta$-catenin signaling pathway and expression of estrogen receptor genes in ovaries of postweaning piglets. J Agric Food Chem 2018; 66:7899-906. https://doi.org/10.1021/acs.jafc.8b02101

10. Reddy KE, Kim M, Kim KH, Ji SY, Lee SD. Effect of commercially purified deoxynivalenol and zearalenone mycotoxins on microbial diversity of pig cecum contents. Anim Biosci 2021;34:243-55. https://doi.org/10.5713/ajas.20.0137

11.Li Y, Zhang B, Huang K, et al. Mitochondrial proteomic analysis reveals the molecular mechanisms underlying reproductive toxicity of zearalenone in MLTC-1 cells. Toxicology 2014; 324:55-67. https://doi.org/10.1016/j.tox.2014.07.007

12. Golli-Bennour EE, Bacha H. Hsp70 expression as biomarkers of oxidative stress: mycotoxins' exploration. Toxicology 2011; 287:1-7. https://doi.org/10.1016/j.tox.2011.06.002

13. Gajęcka M, Rybarczyk L, Jakimiuk E, et al. The effect of experimental long-term exposure to low-dose zearalenone on uterine histology in sexually immature gilts. Exp Toxicol Pathol 2012; 
64:537-42. https://doi.org/10.1016/j.etp.2010.11.009

14. Schoevers EJ, Santos RR, Colenbrander B, Fink-Gremmels J, Roelen BAJ. Transgenerational toxicity of zearalenone in pigs. Reprod Toxicol 2012;34:110-9. https://doi.org/10.1016/j. reprotox.2012.03.004

15. Biehl ML, Prelusky DB, Koritz GD, Hartin KE, Buck WB, Trenholm HL. Biliary excretion and enterohepatic cycling of zearalenone in immature pigs. Toxicol Appl Pharmacol 1993;121:152-9. https://doi.org/10.1006/taap.1993.1140

16. Obremski K. Changes in Th1 and Th2 cytokine concentrations in ileal Peyer's patches in gilts exposed to zearalenone. Pol J Vet Sci 2014;17:53-9. https://doi.org/10.2478/pjvs-2014-0007

17. Maresca M, Fantini J. Some food-associated mycotoxins as potential risk factors in humans predisposed to chronic intestinal inflammatory diseases. Toxicon 2010;56:282-94. https://doi.org/10.1016/j.toxicon.2010.04.016

18. Przybylska-Gornowicz B, Lewczuk B, Prusik M, et al. The effects of deoxynivalenol and zearalenone on the pig large intestine. A light and electron microscopy study. Toxins 2018; 10:148. https://doi.org/10.3390/toxins10040148

19. Przybylska-Gornowicz B, Tarasiuk M, Lewczuk B, et al. The effects of low doses of two Fusarium toxins, zearalenone and deoxynivalenol, on the pig jejunum. A light and electron microscopic study. Toxins 2015;7:4684-705. https://doi.org/ $10.3390 /$ toxins 7114684

20. Cheng Q, Jiang S, Huang L, Ge J, Wang Y, Yang W. Zearalenone induced oxidative stress in the jejunum in postweaning gilts through modulation of the Keap1-Nrf2 signaling pathway and relevant genes. J Anim Sci 2019;97:1722-33. https://doi. org/10.1093/jas/skz051

21.Xiao D, Yuan D, Tan B, Wang J, Liu Y, Tan B. The role of Nrf2 signaling pathway in Eucommia ulmoides flavones regulating oxidative stress in the intestine of piglets. Oxid Med Cell Longev 2019;2019:9719618. https://doi.org/10.1155/2019/ 9719618

22. Kim SK, Yang JW, Kim MR, et al. Increased expression of Nrf2/ARE-dependent anti-oxidant proteins in tamoxifenresistant breast cancer cells. Free Radic Biol Med 2008;45: 537-46. https://doi.org/10.1016/j.freeradbiomed.2008.05. 011

23. Jiang SZ, Yang ZB, Yang WR, et al. Effects of purified zearalenone on growth performance, organ size, serum metabolites, and oxidative stress in postweaning gilts. J Anim Sci 2011;89: 3008-15. https://doi.org/10.2527/jas.2010-3658

24. Jiang SZ, Yang ZB, Yang WR, et al. Effects of feeding purified zearalenone contaminated diets with or without clay enterosorbent on growth, nutrient availability, and genital organs in post-weaning female pigs. Asian-Australas J Anim Sci 2010;23:74-81. https://doi.org/10.5713/ajas.2010.90242

25. Yang L, Wang S, Yang WR, et al. Effects of zearalenone on production performance, serum antioxidant capacity and immune function of weaning gilts. Chin J Anim Nutr 2017;
29:2843-50.

26. Committee on Nutrient Requirements of Swine, National Research Council. Nutrient requirements of swine. 11th ed. Washington, DC, USA: National Academies Press; 2012.

27.Latimer GW; AOAC International. Official methods of analysis of AOAC International. Gaithersburg, MD, USA: AOAC International; 2012.

28. Jiang SZ, Yang ZB, Yang WR, et al. Effect on hepatonephric organs, serum metabolites and oxidative stress in post-weaning piglets fed purified zearalenone-contaminated diets with or without Calibrin-Z. J Anim Physiol Anim Nutr 2012;96:114756. https://doi.org/10.1111/j.1439-0396.2011.01233.x

29. Bradford MM. A rapid and sensitive method for the quantitation of microgram quantities of protein utilizing the principle of protein-dye binding. Anal Biochem 1976;72:248-54. https://doi.org/10.1016/0003-2697(76)90527-3

30.Zhou M, Yang L, Shao M, et al. Effects of zearalenone exposure on the TGF- $\beta 1 / \mathrm{Smad} 3$ signaling pathway and the expression of proliferation or apoptosis related genes of post-weaning gilts. Toxins 2018;10:49. https://doi.org/10.3390/toxins100 20049

31. Rivera A, Agnati LF, Horvath TL, Valderrama JJ, de La Calle A, Fuxe K. Uncoupling protein 2/3 immunoreactivity and the ascending dopaminergic and noradrenergic neuronal systems: relevance for volume transmission. Neuroscience 2006;137:1447-61. https://doi.org/10.1016/j.neuroscience. 2005.05.051

32. Livak KJ, Schmittgen TD. Analysis of relative gene expression data using real-time quantitative PCR and the $2^{-\Delta \Delta C T}$ method. Methods 2001;25:402-8. https://doi.org/10.1006/meth.2001. 1262

33. Tatay E, Espín S, García-Fernández AJ, Ruiz MJ. Oxidative damage and disturbance of antioxidant capacity by zearalenone and its metabolites in human cells. Toxicol In Vitro 2017;45: 334-9. https://doi.org/10.1016/j.tiv.2017.04.026

34.Zheng W, Wang B, Si M, et al. Zearalenone altered the cytoskeletal structure via ER stress- autophagy- oxidative stress pathway in mouse TM4 Sertoli cells. Sci Rep 2018;8:3320. https://doi.org/10.1038/s41598-018-21567-8

35. Bouaziz C, Sharaf el dein O, El Golli E, et al. Different apoptotic pathways induced by zearalenone, T-2 toxin and ochratoxin A in human hepatoma cells. Toxicology 2008;254:19-28. https:// doi.org/10.1016/j.tox.2008.08.020

36. Kouadio JH, Dano SD, Moukha S, Mobio TA, Creppy EE. Effects of combinations of Fusarium mycotoxins on the inhibition of macromolecular synthesis, malondialdehyde levels, DNA methylation and fragmentation, and viability in Caco-2 cells. Toxicon 2007;49:306-17. https://doi.org/10. 1016/j.toxicon.2006.09.029

37. Ren ZH, Deng HD, Wang YC, et al. The Fusarium toxin zearalenone and deoxynivalenol affect murine splenic antioxidant functions, interferon levels, and T-cell subsets. Environ Toxicol 
Pharmacol 2016;41:195-200. https://doi.org/10.1016/j.etap. 2015.12.007

38. Ren Z, Wang Y, Deng H, et al. Deoxynivalenol induces apoptosis in chicken splenic lymphocytes via the reactive oxygen species-mediated mitochondrial pathway. Environ Toxicol Pharmacol 2015;39:339-46. https://doi.org/10.1016/j.etap. 2014.11.028

39. Shi B, Su Y, Chang S, Sun Y, Meng X, Shan A. Vitamin C protects piglet liver against zearalenone-induced oxidative stress by modulating expression of nuclear receptors PXR and CAR and their target genes. Food Funct 2017;8:367587. https://doi.org/10.1039/C7FO01301A

40. Cheng Q, Jiang S, Huang L, et al. Effects of zearalenone-induced oxidative stress and Keap1-Nrf2 signaling pathway-related gene expression in the ileum and mesenteric lymph nodes of post-weaning gilts. Toxicology 2020;429:152337. https:// doi.org/10.1016/j.tox.2019.152337

41.Palliyaguru DL, Chartoumpekis DV, Wakabayashi N, et al. Withaferin a induces nrf2-dependent protection against liver injury: role of keap1-independent mechanisms. Free Radic Biol Med 2016;101:116-28. https://doi.org/10.1016/ j.freeradbiomed.2016.10.003

42. Kubo E, Chhunchha B, Singh P, Sasaki H, Singh DP. Sulforaphane reactivates cellular antioxidant defense by inducing Nrf2/ARE/Prdx6 activity during aging and oxidative stress. Sci Rep 2017;7:14130. https://doi.org/10.1038/s41598-01714520-8

43. Mine Y, Young D, Yang C. Antioxidative stress effect of phos- phoserine dimers is mediated via activation of the Nrf2 signaling pathway. Mol Nutr Food Res 2015;59:303-14. https:// doi.org/10.1002/mnfr.201400381

44. Ye Y, Li J, Yuan Z. Effect of antioxidant vitamin supplementation on cardiovascular outcomes: a meta-analysis of randomized controlled trials. PLoS One 2013;8:e56803. https://doi.org/10. 1371/journal.pone.0056803

45. Xiao Y, Zhang Z, Fu Y, Shan H, Cui S, Wu J. GSTA3 regulates TGF- $\beta 1$-induced renal interstitial fibrosis in NRK-52E cells as a component of the PI3K-Keap1/Nrf2 pathway. J Int Med Res 2019;47:5787-801. https://doi.org/10.1177/030006051 9876796

46.Shanmugam G, Challa AK, Litovsky SH, et al. Enhanced Keap1-Nrf2 signaling protects the myocardium from isoproterenol-induced pathological remodeling in mice. Redox Biol 2019;27:101212. https://doi.org/10.1016/j.redox.2019. 101212

47.Huang Y, Zhou F, Shen C, Wang H, Xiao Y. LBP reduces theinflammatory injuryof kidney in septic rat and regulates the Keap1-Nrf2/ARE signaling pathway. Acta Cir Bras 2019; 34:e20190010000003. https://doi.org/10.1590/s0102-86502019 0010000003

48. Wu CT, Deng JS, Huang WC, Shieh PC, Chung MI, Huang GJ. Salvianolic acid C against acetaminophen-induced acute liver injury by attenuating inflammation, oxidative stress, and apoptosis through inhibition of the Keap1/Nrf2/HO-1 signaling. Oxid Med Cell Longev 2019;2019:9056845. https:// doi.org/10.1155/2019/9056845 